\title{
Modélisation de la décorporation du Pu/Am par le DTPA
}

\author{
P. FRITSCH ${ }^{1}$, A.-L. SÉRANDOUR ${ }^{1}$, O. GRÉMY ${ }^{1}$, B. LE GALL ${ }^{1}$, G. PHAN ${ }^{2}$, \\ N. TSAPIS ${ }^{3}$, E. FATTAL ${ }^{3}$, H. BENECH ${ }^{2}$, J.-R. DEVERRE ${ }^{4}$, N. BLANCHIN ${ }^{5}$, \\ L. GRAPPIN ${ }^{5}$, E. BLANCHARDON ${ }^{6}$, B. BREUSTEDT ${ }^{7}$, J.-L. PONCY ${ }^{1}$
}

(Manuscrit reçu le 23 février 2009, accepté le 29 juin 2009)

RÉSUMÉ Un nouvel outil a été développé pour optimiser l'efficacité des traitements par le DTPA après blessure, sur la base d'une réduction de la dose efficace engagée. Les simulations montrent, notamment, que pour du ${ }^{239} \mathrm{Pu}$ modérément soluble (type $\mathrm{M}$ ), des traitements précoces ( 24 i.v.) étalées sur 4 mois permettent une réduction d'un tiers de la dose, alors que leur prolongement sur 5 ans, avec un intervalle de 2 semaines, peut diminuer la dose d'un facteur 5 . En revanche, pour des composés peu solubles (type $S$ ), l'efficacité des traitements précoces est négligeable et un gain dosimétrique d'un facteur 3 n'est atteint que pour des traitements effectués 2 fois par mois durant 50 ans. Certaines des hypothèses retenues pour la modélisation ont été validées par les résultats d'expérimentations animales récemment publiés. Enfin, la structure d'un nouveau modèle applicable à la fois au Pu et à l'Am est rapportée, structure tenant compte de la décorporation urinaire et fécale de ces actinides et qui pourrait être adapté à différentes posologies et formes galéniques de DTPA.

ABSTRACT Modelling Pu/Am decorporation by DTPA.

A new tool has been developed to optimize DTPA efficacy as concerns reduction of effective dose after ${ }^{239} \mathrm{Pu}$ wound. For example, the simulations show, for moderately soluble compounds (type $M$ ), a 1/3 decrease of effective dose is obtained after repeated early treatment ( 24 i.v. for 4 months), whereas a decrease by a factor 5 can be reached if treatments continue for 5 years at 2 week interval. By contrast, for poorly soluble compounds (type $\mathbf{S}$ ), negligible efficacy is observed after early treatments, and a 3 time decrease of dose is obtained for treatments performed at $\mathbf{2}$ week interval for $\mathbf{5 0}$ years. Some of the hypotheses retained for modelling DTPA decorporation are validated from new experimental data published recently, and structure of a new model which can be applied both to Pu and Am is reported, taking into account urinary and faecal excretion, structure being suitable for different doses of DTPA and using various galenic forms.

Keywords: DTPA / plutonium / americium / inhalation / wound

\footnotetext{
Laboratoire de Radiotoxicologie, CEA/DSV/iRCM/SREIT, BP 12, 91680 Bruyères-le-Châtel, France.

CEA/DSV/iBITEC/SPI, 91191 Gif-sur-Yvette, France.

Université Paris-Sud, UMR CNRS 8612, 92296 Châtenay-Malabry, France.

CEA/DSV/i2BM/SHFJ, 91401 Orsay, France.

CEA/SMT Cadarache, 13108 Saint-Paul-lez-Durance, France.

IRSN/DRPH/SDI/LEDI, BP 17, 92262 Fontenay-aux-Roses Cedex, France.

FZK/GmbH, PO Box 3640, 76021 Karlsruhe, Allemagne.
} 
P. FRITSCH et al.

\section{Introduction}

Après contamination accidentelle par des composés renfermant du $\mathrm{Pu}$, de l'Am et/ou du $\mathrm{Cm}$ qui présentent un très long temps de séjour dans l'organisme, le DTPA peut être administré par différentes voies et sous diverses formes galéniques afin d'augmenter leur décorporation et ainsi limiter les doses engagées. Plusieurs guides ont été mis à jour pour proposer des protocoles de traitements optimisés, en termes de décorporation, tenant compte de l'évolution des connaissances scientifiques et du retour d'expérience (Bhattacharyya et al., 1992 ; Hengé-Napoli et al., 2000 ; Ménétrier et al., 2005).

En France, l'autorisation de mise sur le marché (AMM) du Ca-DTPA en solution fixe la dose quotidienne maximale administrée par i.v. à $1 \mathrm{~g}$, la posologie recommandée étant de 0,5 g ( 15 $\mu \mathrm{mol} / \mathrm{kg})$ (Grappin et Bérard, 2008). En cas de contamination pulmonaire, l'inhalation d'aérosols de poudres sèches de Ca-DTPA peut être effectuée précocement avant l'intervention médicale. Cependant cette forme galénique ne dispose pas d'AMM. Le dépôt pulmonaire maximal recommandé correspond à $60 \mathrm{mg}(2 \mu \mathrm{mol} / \mathrm{kg})$ afin de limiter une toxicité locale. Néanmoins, l'estimation de ce dépôt demeure très approximative et la probabilité d'un dépôt plus faible que celui attendu apparaît relativement grande, du fait de la durée et de la pénibilité d'un traitement effectué à l'aide d'un turbo-inhalateur. En effet, dans le passé, la poudre administrée n'avait pas une granulométrie optimisée pour un dépôt préférentiel au sein du poumon profond. De ce fait, de nouvelles formulations de poudres sèches de Ca-DTPA ont été récemment développées (Tymen et al., 2000 ; Gervelas et al., 2007).

La principale question que se pose le médecin traitant est la suivante : la posologie du DTPA, son mode d'administration et la répétition des traitements sont-ils les mieux adaptés pour limiter les doses engagées en fonction du type de contamination, de son niveau, et de la nature du radiocontaminant? En effet, chaque contamination constitue un cas particulier où des mélanges complexes de particules peu solubles (oxydes d'actinides, particules sur lesquelles des actinides sont adsorbés...), de formes colloïdales (modérément solubles) et de formes monoatomiques (solubles) peuvent être présents. Quand arrêter les traitements ? Quels sont leurs bénéfices en termes de réduction de la dose équivalente au site de la contamination et de la dose efficace ? Le médecin doit répondre à ces questions et informer son patient afin d'optimiser sa démarche thérapeutique.

Plusieurs modèles ont été proposées pour décrire la décorporation du Pu par le DTPA (James et al., 2007 ; Fritsch et al., 2007 ; Breustedt et al., 2009). Cependant, de bons ajustements des données biologiques humaines avec des simulations peuvent être obtenus en appliquant diverses hypothèses concernant la contribution 
respective du site de la contamination, du sang et des liquides interstitiels, et des principaux sites de rétention systémique. Ces modèles ont été élaborés au cas par cas, mais aucun outil n'est aujourd'hui disponible pour simuler les bénéfices dosimétriques associés à différents protocoles de traitements par le DTPA, en fonction des données radiotoxicologiques disponibles.

Trois groupes de travail contribuent actuellement à l'amélioration des protocoles de traitements par le DTPA et de l'interprétation des données radiotoxicologiques. Le groupe MEthodes DOsimétriques de Référence (MEDOR) (Mièle et al., 2007) rédige, dans un cadre de recommandations, un guide destiné aux médecins du travail pour préciser les actions à mener après contamination interne. Le groupe DTPA a réalisé une synthèse des différentes données biologiques recueillies après traitements de travailleurs du CEA et de COGEMA (Grappin et al., 2007) qui a contribuée à l'obtention d'une AMM, en France, de la forme injectable de Ca-DTPA. Il vise maintenant à optimiser les protocoles d'administration du DTPA, pour leur application, au cas par cas. Enfin, à l'échelle européenne, « a COordinated Network for RAdiation Dosimetry » a été créé pour modéliser la décorporation du Pu (Breustedt et al., 2009). Le LRT participe à ces différents groupes, et ensemble, nous avons identifié et hiérarchisé les problématiques à résoudre pour améliorer et justifier les traitements en termes de bénéfices dosimétriques et sanitaires.

Cet article propose un premier outil logiciel couplant le modèle systémique de Leggett pour le Pu (Leggett et al., 2005) avec un modèle décrivant la formation des complexes Pu-DTPA et leur excrétion urinaire qui permettrait d'optimiser les protocoles thérapeutiques sur la base d'une réduction des doses efficaces. Il valide certaines hypothèses retenues pour la modélisation à partir de données animales récemment publiées, et précise la structure d'un nouveau modèle, mieux adapté, qui pourrait être applicable à la fois au $\mathrm{Pu}$ et à l'Am et qui serait transposable à l'homme pour différents protocoles de traitements par diverses posologies et formes galéniques de DTPA.

\section{Intérêt d'un outil pour simuler les bénéfices dosimétriques après traitement}

Différentes simulations ont été effectuées pour illustrer l'intérêt potentiel d'une modélisation afin d'optimiser l'efficacité des traitements par le DTPA en termes de réduction de doses. Elles utilisent les hypothèses retenues pour décrire les données humaines recueillies après une contamination interne par du ${ }^{239} \mathrm{Pu}$, due à une blessure (Fritsch et al., 2007). La formation de complexes Pu-DTPA intéresse une fraction des actinides présents au site de la contamination, dans le sang et les 
liquides interstitiels, dans le foie et le rein urinaire. Une fois formés, ces complexes sont stables et se répartissent dans 3 compartiments non identifiés qui diffèrent par leur cinétique d'excrétion urinaire. La figure 1 montre l'efficacité de la décorporation $\mathrm{du}{ }^{239} \mathrm{Pu}$ modérément ou très peu soluble (type $\mathrm{M}$ ou $\mathrm{S}$ ) en termes de doses efficaces engagées, pour différents protocoles de traitement i.v., précoces, à moyen et long cours. Pour des composés de type $\mathrm{M}$, des traitements précoces étalées sur les 4 premiers mois suivant la contamination (Grappin et al., 2009) permettent de diminuer d'un tiers la dose efficace engagée. La poursuite du traitement par des i.v. répétées 2 fois par mois, à moyen ( 5 ans) ou long cours (50 ans) diminue la dose qui aurait été délivrée sans aucun traitement par un facteur 3 et 5 , respectivement. En revanche, pour des composés de type $\mathrm{S}$, le gain dosimétrique associé à un traitement précoce est quantitativement négligeable. Un traitement à moyen cours ne réduit que de $22 \%$ la dose efficace alors qu'un traitement à long court la réduit d'un facteur 3. Ces résultats montrent, qu'a priori, des protocoles de traitements spécifiques à certains postes de travail peuvent être élaborés, dans la mesure où les propriétés physico-chimiques des radiocontaminants potentiels sont connues. Cependant, si des traitements par i.v. peuvent être envisagés sur une durée de quelques années, notamment pour des niveaux élevés de contamination, leur application à long cours n'est pas réaliste du fait d'une trop grande répétition d'actes médicaux. Une modification du mode d'administration de l'agent chélateur pourrait être alors envisagée et l'administration orale de Zn-DTPA apparaît comme un protocole applicable. L'efficacité de ce type de traitement est peu documentée, mais pour de faibles masses de Pu/Am, certaines données expérimentales indiquent que la décorporation pourrait être similaire à celle obtenue après i.v. (Taylor $e t$ al., 2007).

Différentes données humaines sont compatibles avec certaines des hypothèses retenues pour la modélisation. Il est communément admis que la décorporation du $\mathrm{Pu} / \mathrm{Am}$ est essentiellement effectué par voie urinaire et implique les actinides présents dans les milieux extracellulaires. Cependant, bien que la décorporation maximale soit mesurée dans les urines recueillies au cours des premières 24 heures suivant le traitement, une excrétion urinaire plus élevée que celle observée avant le traitement est constatée durant plusieurs jours. Par ailleurs, il existe une grande variabilité de la décorporation urinaire mesurée au cours du premier jour suivant le traitement qui peut varier d'un facteur 5 à 100 selon l'individu. Ce phénomène reste encore inexpliqué et implique une analyse au cas par cas dans la mesure où il n'est pas possible de distinguer la décorporation systémique et celle du site de la contamination. Cependant, pour un même cas, des traitements différés et suffisamment espacés s'avèrent présenter une efficacité similaire (Grappin et al., 2007). Alors que l'excrétion urinaire du Pu mesurée le premier jour après traitement semble liée à une décorporation des liquides circulants, l'excrétion 


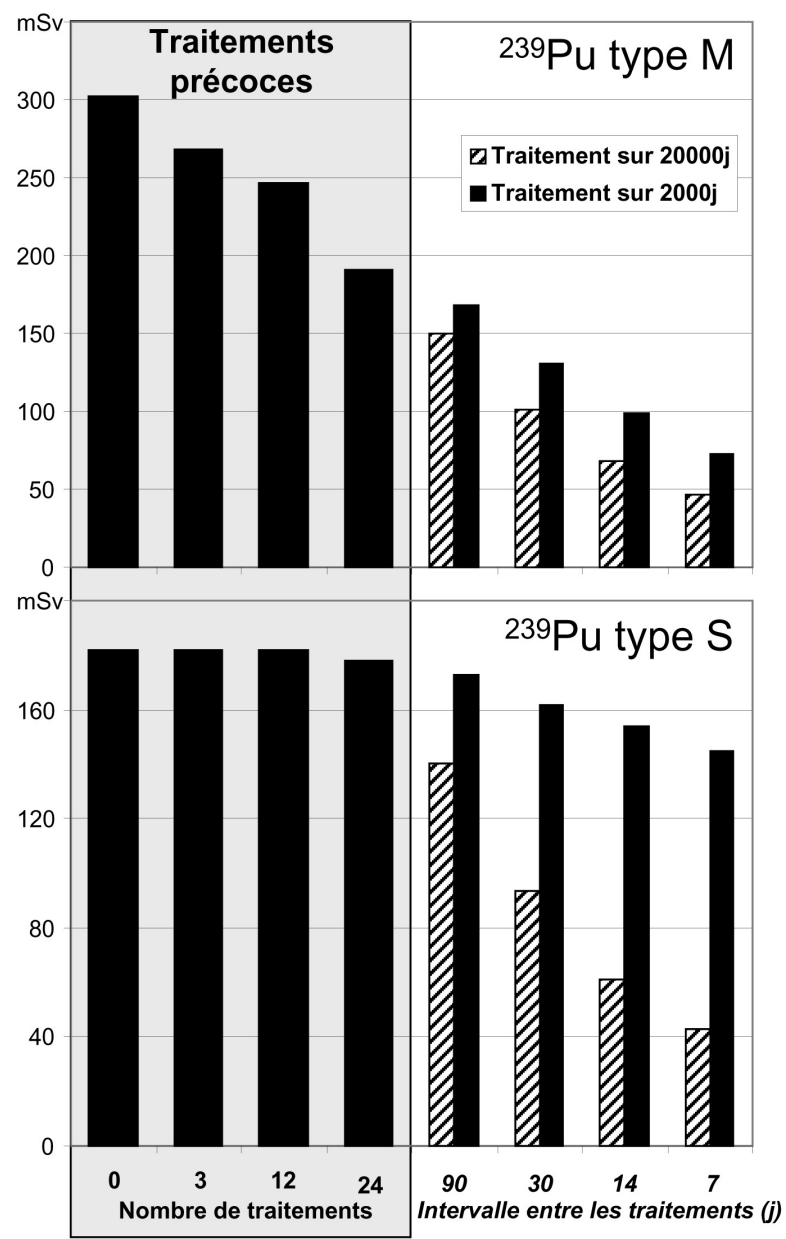

Figure 1 - Influence de différents protocoles de traitement sur les doses efficaces engagées après blessure, en considérant du ${ }^{239} \mathrm{Pu}$ de type $\mathrm{M}$ ou $\mathrm{S}$ et une contamination de $650 \mathrm{~Bq}$. Les traitements précoces correspondent à ceux récemment recommandés (1 i.v. quotidienne les 3 premiers jours, 1 i.v. 2 fois par semaine pendant 3 semaines, et 1 i.v. hebdomadaire pendant 3 mois (Grappin et al., 2009)). Ce traitement peut être poursuivi pendant 2000 ou 20000 jours à intervalle de 7, 14, 30 ou 90 jours. La dose référencée 0 (la première) correspond à celle calculée en absence de traitement.

Influence of DTPA treatment schedule on committed effective doses after ${ }^{239} \mathrm{Pu}$ wound with type $M$ or type $S$ compounds. Early treatments correspond to a schedule recently recommended (1 daily i.v. for the first 3 days, 2 weekly i.v. for 3 weeks, and 1 weekly i.v. for 3 months (Grappin et al., 2009)). This treatment can continue for 2000 or 20000 days at 7, 14, 30 or 90 day interval. Dose at 0 (the first one) corresponds to that obtained without treatment. 
P. FRITSCH et al.

observée les jours suivants pourrait être associée à une décorporation intracellulaire. Différents éléments confortent une telle hypothèse. Des données sur la décorporation de l'Am retenu dans différents tissus ont été obtenues après inhalation de composé de type $M$ et des traitements répétés par i.v. de DTPA qui ont été initiés plusieurs mois après la contamination de 4 individus (Roedler et al., 1989). Une augmentation d'un facteur 65-140 de l'excrétion urinaire de l'actinide et d'un facteur 30-50 de son excrétion fécale peut être mesurée au cours du premier jour suivant un traitement. Le retour à un niveau d'excrétion équivalent à celui observé avant la thérapie n'est obtenu qu'au-delà de 1 mois. Les mesures anthroporadiométriques ont montré une décorporation quasiment totale de l'Am retenu dans le foie, alors qu'aucune augmentation significative n'était observée pour la rétention osseuse. De tels résultats démontrent clairement une décorporation de l'Am retenu au sein des cellules. Une telle conclusion a été également formulée pour le $\mathrm{Pu}$ à partir des excrétions et de la rétention tissulaire post mortem (James et al., 2007). Les données humaines rapportées ici montrent qu'une estimation précise de l'efficacité des traitements doit tenir compte à la fois de l'excrétion urinaire et fécale des actinides qui doit être mesurée à différents temps.

Le modèle que nous avons développé pour une première évaluation des gains dosimétriques demeure empirique. De plus, il est issu de l'analyse d'un cas unique et l'approche est encore générique, sans prise en compte de l'affinité de différents ligands du Pu, de leur concentration et de celle de l'actinide et du DTPA. Par ailleurs, le devenir des complexes Pu-DTPA formés dans l'organisme demeure méconnu. Dans ce contexte, de nouvelles expérimentations ont été initiée afin de valider certaines hypothèses de modélisation en vue du développement d'un outil qui puisse être applicable à différents cas de contamination. Les résultats de ces études sont rapportés dans différentes publications (Sérandour et Fritsch, 2008 ; Sérandour et al., 2008 ; Fritsch et al., 2009a, 2009b).

\section{Devenir biologique des complexes Pu/Am-DTPA chez le rat}

La biocinétique du Pu/Am a été étudiée après i.v. de Pu/Am-DTPA ou de Pu/Amcitrate en solution. Après i.v. de Pu/Am-citrate, le squelette constitue le principal compartiment de rétention, et au-delà de 2 jours, l'excrétion urinaire quotidienne ne représente qu'environ $0,1 \%$ de l'activité corporelle. En revanche, durant la première semaine suivant une i.v. de Pu-DTPA, une activité sensiblement égale est mesurée dans le squelette, le foie et les reins, l'excrétion urinaire de l'actinide représentant environ $10 \%$ de la rétention corporelle. La biocinétique de l'Am a été suivie durant 17 jours après contamination. À ce temps, la rétention maximale est observée dans le squelette $(0,1 \%$ de l'activité injectée pour l'Am-DTPA et $50 \%$ 


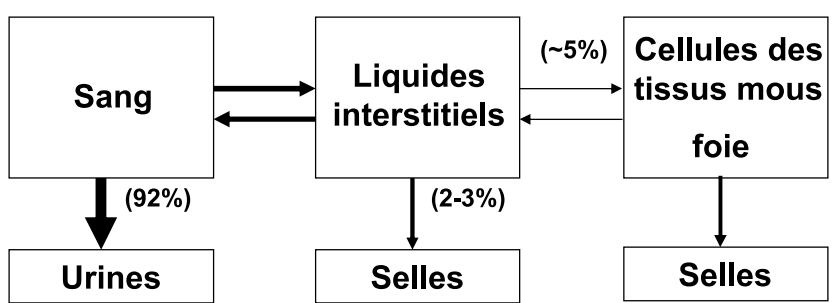

Figure 2 - Structure d'un modèle pour décrire le devenir biologique du DTPA. Entre parenthèses sont figurées la part respective des rétentions et excrétions. On suppose un devenir analogue pour les complexes actinides-DTPA et ceux formés avec d'autres éléments. Un couplage avec un modèle décrivant le devenir systémique des actinides est possible en considérant des compartiments intracellulaires similaires.

Structure of a model to describe biological behaviour of DTPA. Relative contribution in retentions and excretion are provided between brackets. A similar behaviour is assumed for actinides-DTPA and complexes formed with other elements. This model can be easily coupled with that describing systemic actinide behaviour if similar intracellular compartments are taken into account.

pour l'Am-citrate). Dans le cas de l'Am-DTPA, le squelette renferme 2 à 3 fois plus d'activité que celle mesurée dans le foie et les reins, alors que pour l'Amcitrate, les rapports de la rétention os/foie et foie/reins sont proches de 10. Au-delà de 1 jour suivant l'i.v. d'Am-DTPA, l'excrétion urinaire cumulée de l'actinide représente 7 à $8 \%$ de l'activité injectée alors qu'une excrétion fécale voisine de $1 \%$ n'est observée que durant les 2 premiers jours.

L'ensemble de ces données permet de formuler les conclusions suivantes qui sont schématisées dans la figure 2 :

- les complexes actinides-DTPA peuvent être considérés comme stables au sein des différents compartiments de l'organisme (une dissociation voisine de $0,1 \%$ des complexes est observée dans le cas de l'Am-DTPA),

- environ $90 \%$ des complexes présents dans les compartiments extracellulaires sont rapidement excrétés via les urines et $2 \%$ via les selles,

- au moins $5 \%$ des complexes actinide-DTPA sont internalisés au sein des cellules des tissus mous ce qui ralentit la décorporation. Cette conclusion s'appliquerait également au DTPA complexant d'autres métaux.

\section{Origine du Pu/Am extracellulaire décorporé par le DTPA}

L'origine du Pu/Am décorporé au sein des compartiments extracellulaires a été étudiée après i.v. de complexes actinide-citrate suivie, après 1 heure, par une i.v. d'une solution de Ca-DTPA. Au moment du traitement, $50 \%$ du Pu est encore présent dans le sang alors qu'il ne reste que moins de $3 \%$ de l'Am. Ce dépôt très 
rapide de l'Am au sein des tissus de rétention est expliqué du fait d'une plus faible affinité de la transferrine (Tf) pour cet actinide $\left(\log _{10} \mathrm{Ka}: \mathrm{Pu} 22,5\right.$, Am 10,4 (IUPAC, 2004 ; Ansoborlo et al., 2006)). Ainsi, à l'équilibre, plus de $90 \% \mathrm{du} \mathrm{Pu}$ plasmatique est sont forme de $\mathrm{Pu}-\mathrm{Tf}$, alors que cette valeur serait de l'ordre de $30 \%$ pour l'Am (Turner et Taylor, 1968). Pour la modélisation, les autres formes chimiques du $\mathrm{Pu} / \mathrm{Am}$ sont assimilées à des complexes de faibles poids moléculaire qui sont ultrafiltrables (UF).

In vitro, pour une dose équivalente à $30 \mu \mathrm{mol} / \mathrm{kg}$, seuls $5 \%$ du Pu-Tf forment des complexes Pu-DTPA, alors qu'in vivo, l'ultrafiltrabilté plasmatique dans l'heure qui suit le traitement est également voisine de $5 \%$. Ainsi, dans nos conditions expérimentales, le $\mathrm{Pu}$-Tf n'est pas la principale forme chimique directement impliqué dans la décorporation. Des ligands intermédiaires ayant une plus faible affinité pour l'actinide qui forment des complexes avant l'internalisation des actinides dans les cellules ou avant leur dépôt dans la matrice osseuse minéralisée apparaissent impliqués. L'influence de la concentration molaire du Pu dans le plasma de rat sur la formation de complexes Pu-DTPA mesurée par ultrafiltration a été étudiée dans une gamme allant de 1 à $35 \mathrm{nM}$. Les résultats obtenus montrent que le déplacement du Pu par l'agent chélateur répond à la loi d'action de masse et donc que la modélisation doit tenir compte de l'évolution des concentrations extracellulaires de l'agent chélateur et des actinides ainsi que de la nature, la quantité et l'affinité de leurs ligands. Un bon ajustement des données biocinétiques recueillies après i.v. de Pu-citrate et durant le premier jour après traitement peut être obtenu en retenant les hypothèses suivantes :

- des échanges permanents s'effectuent entre le Pu lié à Tf, UF et aux autres ligands extracellulaires,

- pour chaque type de ligands un déplacement total de l'actinide est obtenu audelà d'un rapport DTPA/actinide donné,

- en-dessous de ce rapport seuil, le déplacement diminue de manière linéaire avec une pente spécifique à chaque type de ligand (ceci permet de tenir compte à la fois de la quantité de ligand et de son affinité pour l'actinide).

La figure 3 schématise la structure du modèle retenu. Cette démarche simplifiée permet d'obtenir des variations de l'efficacité de la décorporation en fonction de la dose de Ca-DTPA qui apparaissent compatibles avec les données expérimentales. Il ne semble donc pas utile de tenir compte de la substitution du Ca par d'autres cations tel $\mathrm{Zn}^{++}$qui limite l'efficacité de la décorporation extracellulaire du Pu par le DTPA (FDA, 2002). Par la suite, les paramètres de ce modèle seront ajustés pour décrire la décorporation de l'Am en tenant compte de l'affinité de Tf pour cet élément. 


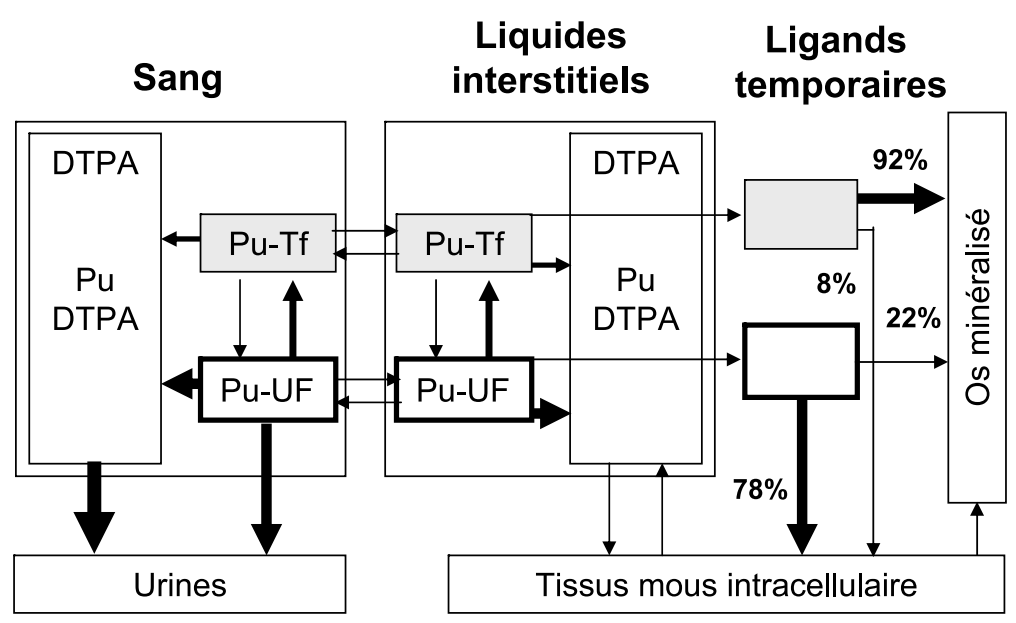

Figure 3 - Modèle simplifié pour décrire le devenir systémique du Pu et sa décorporation extracellulaire par le DTPA. Il s'agit d'un modèle hybride avec des compartiments dont les échanges sont caractérisés par des constantes de temps et des distributions de rétention qui varient selon l'origine des dépôts. Les valeurs en \% correspondent à ces distributions et l'épaisseur des flèches visualise la rapidité des échanges. Ce modèle pourrait être transposé à l'Am en tenant compte de l'affinité de Tf pour cet actinide.

Simplified model to describe systemic behaviour of Pu and its decorporation by DTPA. It is a hybrid model mixing compartments characterized by daily rate of exchange and distribution of retentions which varies depending on the origin of deposits. Such an approach allows us to limit the number of compartments to take into account. Values in \% correspond to these distributions and arrow thickness reflects the rate of exchanges. This model could be transposed to Am after taking into account Tf affinity for this actinide.

\section{La décorporation intracellulaire du Pu/Am par le DTPA}

A priori, les hypothèses à retenir pour décrire la décorporation intracellulaire $\mathrm{du}$ $\mathrm{Pu} / \mathrm{Am}$ seraient beaucoup plus nombreuses que celles formulées précédemment.

Dans le but de distinguer, in vivo, la décorporation extra et intracellulaire du $\mathrm{Pu}$ par le DTPA nous avons comparé la cinétique de l'excrétion urinaire du Pu et sa rétention tissulaire après contamination systémique par du Pu-citrate, précédée, 24 heures avant, ou suivie, 1 heure après, par une administration intraveineuse de Ca-DTPA à différentes doses. Dans le cas d'un prétraitement, la quantité de DTPA présente dans le sang au moment de la contamination correspond à $0,025 \%$ de la quantité administrée. Elle apparaîtrait donc insuffisante pour permettre une décorporation significative du $\mathrm{Pu}$ présent au sein des compartiments extracellulaires. De plus, le DTPA n'est plus sous sa forme injectée car le Ca initialement complexé a été substitué par d'autres éléments formant des complexes 


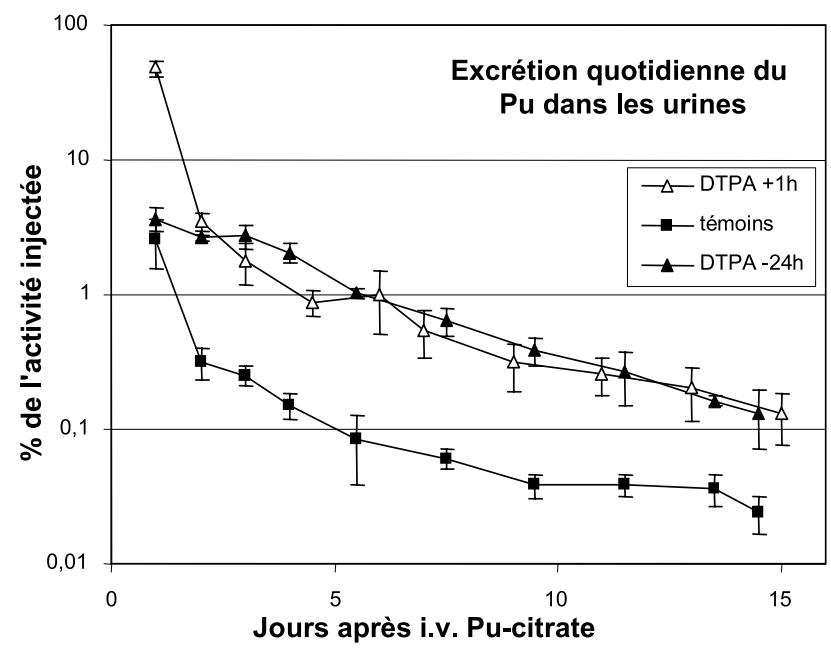

Figure 4 - Comparaison de l'excrétion urinaire quotidienne du Pu après i.v. de Pu-citrate chez des rats non traités ou traités par une i.v. de Ca-DTPA (dose de $30 \mu \mathrm{mol} / \mathrm{kg}$ ) administré 24 heures avant, ou 1 heure après la contamination. Valeurs moyennes \pm écart type. D'après Fritsch et al., 2009 .

Comparison of daily urinary excretion of Pu after Pu-citrate i.v. in rats untreated, or treated by Ca-DTPA i.v. (dose 30 mmol/kg) administered 24 hours before or 1 hour after contamination. Mean values \pm standard deviation. From Fritsch et al., 2009 a.

plus stables. La figure 4 compare la cinétique de l'excrétion urinaire chez des rats non traités, et pré ou post traités à la dose de $30 \mu \mathrm{mol} / \mathrm{kg}$.

À cette dose, les mesures de rétention corporelle effectuées après 14 jours montrent qu'un prétraitement est moitié moins efficace qu'un traitement effectué 1 heure après la contamination. Pour un prétraitement, la cinétique de l'excrétion urinaire du $\mathrm{Pu}$ peut être décrite par une simple fonction exponentielle ayant une période de 2,3 jours. Ainsi, contrairement aux compartiments extracellulaires, la quantité de DTPA présente dans les compartiments intracellulaires peut être considérée comme stable durant plusieurs heures après le traitement. Une simplification pourrait donc être retenue pour la modélisation qui consiste à supposer que la formation des complexes actinides DTPA intracellulaire est proportionnelle au rapport de concentration DTPA/actinides, et comme précédemment, une pente serait spécifique à chaque compartiment.

Une cinétique urinaire similaire est observée pour un prétraitement effectué à la dose de $300 \mu \mathrm{mol} / \mathrm{kg}$. Cependant, pour cette dose, une décorporation quasitotale du foie est observée qui est associée à une forte augmentation du Pu excrété 


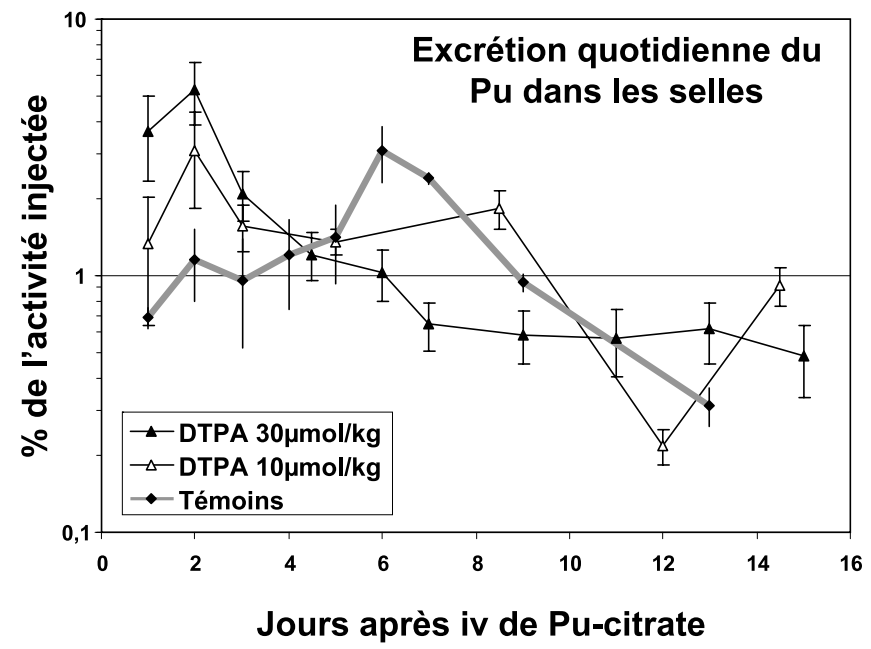

Figure 5 - Comparaison de l'excrétion fécale quotidienne du Pu après i.v. de Pu-citrate chez des rats non traités ou traités par i.v. d'une solution de Ca-DTPA (dose de 10 ou $30 \mu \mathrm{mol} / \mathrm{kg}$ ) effectuée 1 heure après la contamination. Valeurs moyennes pour 3 animaux \pm écart type (données non publiées).

Comparison of daily faecal excretion of Pu after Pu-citrate i.v. in rats untreated, or treated by i.v. of Ca-DTPA solution (dose of 10 or $30 \mu \mathrm{mol} / \mathrm{kg}$ ) performed 1 hour after contamination. Mean values for 3 animals \pm standard deviation (unpublished data).

dans les selles recueillies durant les 3 premiers jours. Ce phénomène pourrait visualiser une décorporation hépatique intracellulaire par voie biliaire.

Deux modèles de contamination ont été utilisés pour mieux caractériser la décorporation hépatique du $\mathrm{Pu} / \mathrm{Am}$ : l'i.v. de complexes citrate ou phytate. Ces derniers complexes se comportent comme des formes colloïdales, $90 \%$ de l'activité étant retenus dans le foie en moins d'une heure. Les résultats obtenus montrent qu'il existe 2 modes de décorporation hépatique : une décorporation via le sang et l'urine qui est prépondérante après i.v. de Pu-phytate, et une décorporation via la bile qui est clairement mise en évidence après i.v. d'Amcitrate. En fait, chez le rat, la décorporation par voie biliaire correspond à une accélération d'un processus naturel d'épuration car, après quelques jours, l'excrétion cumulée devient inférieure à celle mesurée chez les animaux non traités. La figure 5 montre qu'une même conclusion peut être formulée pour le Pu. Ce phénomène pourrait être spécifique aux rongeurs dans la mesure où des expérimentations ciblées chez le babouin montrent une décorporation fécale similaire à celle observée chez l'homme où la décorporation induite par le DTPA est supérieure à la décorporation spontanée. D'autres expérimentations ont 

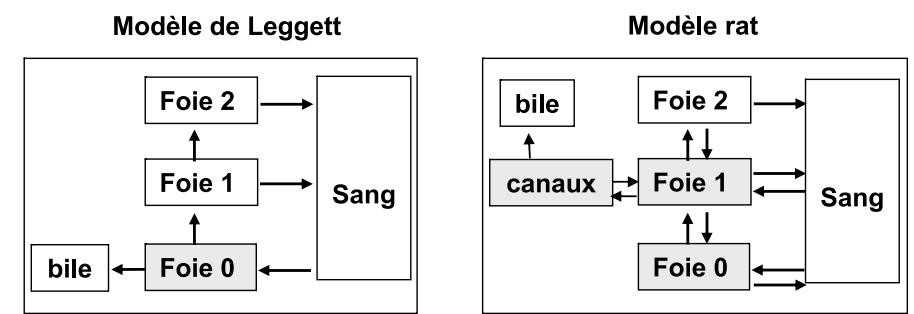

Figure 6 - Comparaison de la structure du modèle hépatique proposé chez l’homme pour le Pu (Leggett et al., 2005) avec celui retenu pour décrire la décorporation hépatique via le sang et la bile. Les compartiments hépatiques décorporables par le DTPA apparaissent en grisé. Les différences rongeur/primate peuvent être décrites en modifiant les échanges entre les canalicules biliaires et Foie 0.

Comparison of hepatic model proposed in human for Pu (Leggett et al., 2005) with that retained to describe urinary and faecal decorporation. Compartments which can be decorporated appear in gray. Differences observed between rodent and primate can be described after variation of the rate of exchanges between biliary ducts and liver 0.

montré, pour une même dose, que l'encapsulation de DTPA au sein de liposomes permet une très forte augmentation de l'efficacité de la décorporation des actinides comparativement à l'administration i.v. d'une solution. L'ensemble des résultats obtenus indique que pour le foie, l'efficacité de la décorporation est fonction de la quantité de DTPA internalisé dans les cellules et qu'elle devient proche de $100 \%$ pour des rapports en concentration de l'agent chélateur sur les actinides supérieurs à 1000 .

La structure du modèle hépatique proposé par Leggett apparaît peu adaptée pour décrire la décorporation du Pu/Am qui implique à la fois une excrétion urinaire et fécale. Un autre modèle est donc proposé (Fig. 6) qui pourrait être plus physiologique dans la mesure où il distingue des compartiments pouvant être associés aux cellules de Kupfer (foie 0) et aux hépatocytes (foie 1 et 2).

\section{Décorporation pulmonaire du Pu/Am}

Différentes études ont montré une décorporation pulmonaire quasi-totale de formes monoatomiques de Pu/Am après administration locale de DTPA par inhalation d'aérosols, même lorsque le traitement était initié plusieurs semaines après la contamination (Stradling et al., 1984 ; Stather et al., 1985). En fait, tout comme pour le foie, l'efficacité de la décorporation est fonction du rapport en concentration pulmonaire du DTPA/actinides et, pour de faibles masses d'actinides, une décorporation similaire peut être mesurée après i.v. de DTPA ou après addition de Zn-DTPA à l'eau de boisson (Taylor et al., 2007). 


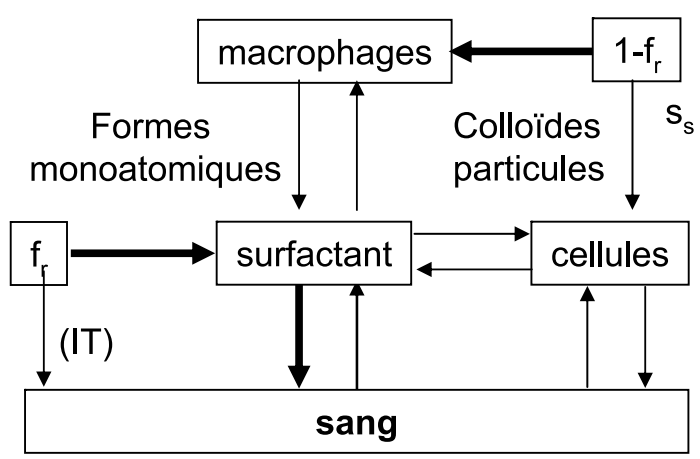

Figure 7 -Modèle propose pour decrire la décorporation de différentes formes chimiques de Pu/Am retenues au sein des poumons. Après instillation intratrachéale (IT), une fraction de $f_{r}$ peut passer directement vers le sang du fait d'une perturbation de la structure du surfactant. Ce phénomène ne serait pas observé après inhalation. Il est possible de tenir compte d'une décorporation des colloüdes en relation avec la quantité de DTPA présente dans les poumons. Model describing decorporation of different chemical forms of Pu/Am retained in the lungs. After intratracheal instillation (IT) a fraction of $f_{r}$ can be transfer directly to the blood due to modification of surfactant structure. Such a phenomenon should not occur after inhalation. Decorporation of colloids can be described in relation with the DTPA content of the lungs.

L'autoradiographie permet de distinguer différentes formes physico-chimiques de $\mathrm{Pu} / \mathrm{Am}$ retenues au sein des poumons. Les formes monoatomiques sont visualisées comme des traces alpha isolées réparties uniformément au sein du parenchyme pulmonaire. Les formes colloïdales apparaissent comme des spots ne présentant pas une symétrie nette par rapport à un point alors que les particules d'oxyde de $\mathrm{Pu} / \mathrm{Am}$ présentent une telle symétrie. Oxydes et collö̈des apparaissent souvent associés à des macrophages alvéolaires. L'incubation de coupes de poumons dans une solution de DTPA permet de distinguer les formes monoatomiques d'Am (extraites) et de $\mathrm{Pu}$ (non extraites), les formes colloïdales d'actinides sont partiellement extraites, alors que les oxydes ne le sont pas. À partir de ces résultats un modèle est proposé (Fig. 7) qui permet, notamment, de décrire le devenir du $\mathrm{Pu}$ après inhalation d'oxydes ou instillation intratrachéale d'une solution de Pu-nitrate à des masses où une formation significative de colloïdes est observée.

Une grande partie des colloïdes apparaissent décorporables par le DTPA dans la mesure où la quantité de DTPA présente dans les poumons serait suffisante. Ceci justifierait l'administration locale de l'agent chélateur après inhalation de masses significatives de Pu/Am de type M. En revanche, pour de faibles masses, et pour des composés de type $\mathrm{S}$, le gain dosimétrique de ce type de traitement serait très faible, similaire à celui obtenu après i.v. de Ca-DTPA. 
P. FRITSCH et al.

\section{Conclusion}

Une modélisation de la décorporation du Pu/Am par le DTPA apparaît aujourd'hui possible qui pourrait s' appliquer à différents types de contamination. Elle implique la prise en compte de nombreux paramètres spécifiques aux radiocontaminants, à la localisation et à la spéciation des actinides au sein de l'organisme, et aux protocoles de traitement qui ne peut se faire sans hypothèses simplificatrices. Ainsi, le modèle proposé est un modèle hybride qui fait appel à des compartiments dont les échanges sont décrits par des constantes de temps, mais aussi à des fractions qui permettent de définir la répartition de différents dépôts ou de différentes formes chimiques. Dans la pratique, une stratégie thérapeutique logique se dessine qui consiste, dans un premier temps, à optimiser l'efficacité des traitements précoces sur des critères relatifs aux niveaux de contamination et aux formes physico-chimiques impliquées. L'analyse de différentes données radiotoxicologiques (mesure de l'activité au site de contamination et des excrétions urinaires et fécales) pourrait alors permettre d'évaluer le comportement du radiocontaminant (type $\mathrm{M}$ ou $\mathrm{S}$ ). Ensuite, et si nécessaire, après arrêt des traitements, il faut définir les analyses utiles à une évaluation plus précise des paramètres de dissolution. Enfin, en fonction de la valeur de ces paramètres, des thérapies peuvent être entreprises à moyen ou long cours. Dans ce cadre, de nouvelles recherches galéniques s'imposent pour assurer la faisabilité de traitements répétitifs.

En conclusion, ce travail souligne l'intérêt de la connaissance des mécanismes impliqués dans la décorporation des actinides par le DTPA et que seuls des critères dosimétriques doivent être pris en compte pour justifier et optimiser les traitements.

Remerciements. Ce travail a été partiellement financé dans le cadre des accords CEA / AREVA (PIC D13). Cet article fait référence à certaines données non encore publiées qui peuvent être accessibles en contactant paul.fritsch@cea.fr.

\section{RÉFÉRENCES}

Ansoborlo E., Prat O., Moisy P., Den Auver C., Guilbaud P., Carriere M., Gouget B., Duffield J., Doizi D., Vercouter T., Moulin C., Moulin V. (2006) Actinide speciation in relation with biological processes, Biochimie 88, 1605-1618.

Bhattacharyya M.H., Breitenstein B.D., Métivier H., Muggenburg B.A., Stradling G.N., Volf V. (1992) Guidebook for the treatment of accidental internal contamination of workers, Nuclear Technology Publishing, Ashford.

Breustedt B., Blanchardon E., Fritsch P., Giussani A., Lopez M.A., Luciani A., Malatova I., Nosske D., Piechowski J., Schimmelpfeng J., Sérandour A.-L. (2009) Biokinetic modeling of DTPA decorporation therapy: the CONRAD approach, Radiat. Prot. Dosim. 134, 38-48. 
FDA (2002) Food and Drug Administration, Guidance for industry on pentetate calcium trisodium and pentetate zinc trisodium for treatment of internal contamination with plutonium, americium or curium; availability, Federal Register 68, 53984-53988.

Fritsch P., Grappin L., Guillermin A.M., Fottorino R., Ruffin M., Mièle A. (2007) Modelling of bioassay data from a Pu wound treated by repeated DTPA perfusions: biokinetics and dosimetric approaches, Radiat. Prot. Dosim. 127, 120-124.

Fritsch P., Sérandour A.L., Grémy O., Phan G., Tsapis N., Abram M.C., Renault D., Fattal E., Benech H., Deverre J.R., Poncy J.L. (2009a) Simplified structure of a new model to describe urinary excretion of $\mathrm{Pu}$ after systemic, liver or pulmonary contamination of rats associated with CaDTPA treatments, Radiat. Res. 171, 674-686.

Fritsch P., Sérandour A.L., Grémy O., Phan G., Tsapis N., Fattal E., Benech H., Deverre J.R., Poncy J.L. (2009b) Structure of a single model to describe plutonium and americium decorporation by DTPA treatment, Health Phys. (soumis).

Gervelas C., Sérandour A.L., Geiger S., Grillon G., Fritsch P., Taulelle C., Le Gall B., Benech H., Deverre J.R., Fattal E., Tsapis N. (2007) Direct lung delivery of a dry powder formulation of DTPA with improved aerosolization properties: effect on lung and systemic decorporation of plutonium, J. Control. Release 118, 78-86.

Grappin L., Bérard P., Ménétrier F., Carbone L., Courtray C., Castagneux X., Le Golf J.P., Neron M.O., Piechowski J. (2007) Treatment of actinide exposures: a review of Ca-DTPA injections inside CEA-COGEMA plants, Radiat. Prot. Dosim. 127, 435-439.

Grappin L., Bérard P. (2008) Autorisation de mise sur le marché du Ca-DTPA, Radioprotection 43, 465-466.

Grappin L., Legoff J.P., Carbone L., Courtay C., Agrinier A.L., Animat M., Amabile J.C., Florin A., André F. (2009) Traitement par le Ca-DTPA des contaminations internes par plutonium et américium : Recommandations pour la rédaction de protocoles dans les centres CEA et AREVA, Radioprotection 44, 447-461.

Hengé-Napoli M.H., Stradling G.N., Taylor D.M. (2000) Decorporation of radionuclides from the human body, Radiat. Prot. Dosim. 87, 11.

IUPAC (2004) Stability constants database, Academic Software, Timble, U.K.

James A.C., Sasser L.B., Stuit D.B., Glover S.E., Carbaugh E.H. (2007) USTUR whole body case 0269: demonstrating effectiveness of I.V. Ca-DTPA for Pu, Radiat. Prot. Dosim. 127, 114-119.

Leggett R.W., Eckerman K.F., Khokhryakov V.F., Suslova K.G., Krahenbuhl M.P., Miller S.C. (2005) Mayak worker study: an improved biokinetic model for reconstructing doses from internally deposited plutonium, Radiat. Res. 164, 111-122.

Ménétrier F., Grappin L., Raynaud P., Courtay C., Wood R., Joussineau S., List V., Stradling G.N., Taylor D.M., Bérard P., Morcillo M.A., Rencova J. (2005) Treatment of accidental intakes of plutonium and americium: guidance notes, Appl. Radiat. Isot. 62, 829-846.

Mièle A., Blanchin N., Raynaud P., Quesne B., Giraud J.M., Fottorino R., Bérard P., Ansoborlo E., Franck D., Blanchardon E., Challeton-de Vathaire C., Lebaron-Jacobs L., Poncy J.L., Piechowski J., Fritsch P. (2007) MEDOR, a didactic tool to support interpretation of bioassay data after internal contamin Radiat. Prot. Dosim. 127, 350-355.

Roedler H.D., Noßke D., Ohlenschäger L., Schieferdecker H., Doerfel H., Renz K. (1989) Incorporation of ${ }^{241} \mathrm{Am}$ : effectiveness of late chelation therapy, Radiat. Prot. Dosim. 26, 377379.

Sérandour A.L., Grémy O., Fréchou M., Renault D., Poncy J.L., Fritsch P. (2008) In vitro and in vivo assessment of plutonium speciation and decorporation in blood and target retention tissues after a systemic contamination followed by an early DTPA treatment, Radiat. Res. 170, 208-215.

Sérandour A.L., Fritsch P. (2008) Pulmonary retention of actinides after dissolution of $\mathrm{PuO}_{2}$ aerosols: interest in modelling DTPA decorporation, Radioprotection 43, 239-254. 
Stather J.W., Stradling G.N., Gray S.A., Moody J., Hodgson A. (1985) Use of DTPA for increasing the rate of elimination of plutonium-238 and americium-241 from rodents after their inhalation as the nitrates, Hum. Toxicol. 4, 573-582.

Stradling G.N., Stather J.W., Sumner S.A., Strong J.C., Lennox A.M., Ham S.E. (1984) Decorporation of inhaled plutonium nitrate from hamsters using Zn-DTPA, Health Phys. 46, 919-924.

Taylor D.M., Hodgson S.A., Stradling N. (2007) Treatment of human contaminations with plutonium and americium: would orally administered Ca- or Zn-DTPA be effective? Radiat. Prot. Dosim. 127, 469-471.

Turner G.A., Taylor D.M. (1968) The transport of plutonium, americium and curium in the blood of rats, Phys. Med. Biol. 13, 535-546.

Tymen H., Schoulz D., Caire-Maurisier A.M., Chevrier F., Curet P.M. (2000) Emergency treatment of pulmonary contaminations by transuranium elements. A new galenic form of $\mathrm{Na}_{3} \mathrm{CaDTPA}$, Radioprotection 35, 473-485. 